\title{
Puntos intermedios de la prueba de tolerancia oral a la glucosa en pacientes adultos con fibrosis quística
}

\section{Intermediate points of the oral glucose tolerance test in adult patients with cystic fibrosis}

\author{
Ezequiel Baran ${ }^{1 *}$, Alejandra C. Godoy², Julieta A. González², Ma. Virginia D’Ascenzo ${ }^{3}$, Guido Copparoni²,
} Marina Y. Zubillaga ${ }^{2}$ y Amelia J. Rivera ${ }^{2}$

${ }^{1}$ Servicio de Neumonología; ${ }^{2}$ Servicio de Laboratorio; ${ }^{3}$ Servicio de Gastroenterología. Hospital Interzonal General de Agudos Dr. Rodolfo Rossi, La Plata, Argentina

\section{RESUMEN}

Objetivos: Evaluar la utilidad de los puntos 30, 60 y 90 minutos de la prueba de tolerancia oral a la glucosa (PTOG) en pacientes con fibrosis quística (FQ) y la correlación de las alteraciones del metabolismo hidrocarbonado con la función pulmonar y el estado nutricional. Materiales y métodos: Estudio observacional prospectivo transversal. Se incluyeron pacientes mayores de 16 años con FQ. Se registró el volumen espiratorio forzado en el primer segundo $\left(V_{1} F_{1}\right)$, el índice de masa corporal (IMC) y se realizó la PTOG extendida. Se determinó el porcentaje de pacientes con intolerancia a la glucosa (ITG), tolerancia a la glucosa indeterminada (INDET), glucemia basal alterada (GBA) y tolerancia normal a la glucosa (TGN). Se comparó estadísticamente $V_{E F}, V_{1} \%$ e IMC entre los pacientes con y sin alteraciones del metabolismo hidrocarbonado. Resultados: Se estudiaron 28 pacientes: 14 (50\%) TGN, 3 (10.7\%) GBA, 7 (25\%) ITG y 4 (14.3\%) INDET. Evaluación de VEF : se encontró diferencia significativa para ITG respecto a TGN; no se encontró diferencia significativa para INDET y GBA respecto a TGN. Evaluación de VEF\% e IMC: no se encontraron diferencias significativas entre los grupos. Conclusiones: La PTOG extendida detectó un $14.3 \%$ de INDET, los cuales se hubiesen perdido al realizar la PTOG clásica. Estos pacientes no presentaron alteraciones en $\mathrm{VEF}_{1}$ e IMC respecto a TGN. A los cuatro años de seguimiento dichos pacientes presentaron diabetes relacionada a la FQ.

Palabras clave: Test de tolerancia oral a la glucosa. Fibrosis quística. Adulto.

\section{ABSTRACT}

Objectives: To assess the usefulness of the 30,60 and 90 minutes points of the oral glucose tolerance test (OGTT) in patients with cystic fibrosis (CF) and the correlation of alterations in hydrocarbon metabolism with lung function and nutritional condition. Materials and methods: Observational prospective cross-sectional study. Patients older than 16 years with CF were included. Forced espiratory volume was recorded in the first second $\left(F E V_{1}\right)$, Body Mass Index (BMI) and extended OGTT was performed. The percentage of patients with intolerance glucose (IGT), indeterminate glucose tolerance (INDET), altered basal glycemia (ABG) and normal glucose tolerance (NGT) was determined. $\mathrm{FEV}_{1}, \mathrm{FEV}_{1} \%$ and $\mathrm{BMI}$ were statistically compared between patients with and without alterations in hydrocarbon metabolism. Results: 28 patients were studied: 14 (50\%) NGT, 3 (10.7\%) AGB, 7 (25\%) IGT and 4 (14.3\%) INDET. FEV evaluation: significant difference was found for IGT with respect to NGT; No significant difference was found for INDET and AGB with respect to NGT. $\mathrm{FEV}_{1} \%$ and $\mathrm{BMI}$ evaluation: no significant differences were found between the groups. Conclusions: Extended PTOG detected $14.3 \%$ of INDET, which would have been lost when performing classical GTT. These patients had no changes in $\mathrm{FEV}_{1}$ and $\mathrm{BMI}$ compared to NGT. At 4 years of follow-up, these patients had diabetes related to cystic fibrosis.

Key words: Oral glucose tolerance test. Cystic fibrosis. Adult.
Correspondencia:

*Ezequiel Baran

E-mail: ezebarandgmail.com
Fecha de recepción: 08-02-2021

Fecha de aceptación: 16-06-2021

DOI: 10.24875/ALAD.21000004
Disponible en internet: 29-09-2021 Rev ALAD. 2021;11:63-73

2248-6518 / @ 2021 Asociación Latinoamericana de Diabetes. Publicado por Permanyer. Este es un artículo open access bajo la licencia CC BY-NC-ND (http://creativecommons.org/licenses/by-nc-nd/4.0/). 


\section{INTRODUCCIÓN}

La fibrosis quística (FQ) es una enfermedad genética, de herencia autosómica recesiva, caracterizada por una mutación en el gen codificador de la proteína transmembrana reguladora de la conductividad de la FQ (CFTR)' ${ }^{1}$. Las mutaciones en el gen CFTR resultan en una pérdida variable del transporte de cloro en células epiteliales, causando disfunción de las glándulas de secreción exocrina ${ }^{2,3}$. Las personas afectadas producen una mucosidad espesa y viscosa que provoca una obstrucción de los conductos de los órganos donde se localiza, siendo el páncreas y los pulmones los órganos más comprometidos, aunque se trata de una entidad multisistémica ${ }^{4}$.

En las últimas décadas la supervivencia de los pacientes con FQ ha mejorado significativamente gracias a los avances en los cuidados médicos respiratorios y nutricionales ${ }^{1,5}$. Sin embargo, han emergido comorbilidades, siendo una de las más frecuentes la diabetes relacionada a FQ (DRFQ) 1,2,5. La prevalencia de DRFQ aumenta con la edad, llegando a ser de hasta un $50 \%$ a los 30 años $^{6-10}$.

En general, en estos pacientes existe fibrosis pancreática severa e insuficiencia exocrina que frecuentemente se asocia a disfunción endocrina. Esta puede llevar a una alteración del metabolismo hidrocarbonado e incluso evolucionar a diabetes ${ }^{11}$. Se sabe que el principal factor fisiopatogénico es el déficit de insulina asociado a la disfunción de la célula beta, aunque también influyen otros factores como la resistencia a la insulina, la alteración del funcionamiento de otras hormonas pancreáticas, la alteración del eje enteroinsular y el aclaramiento de la insulina. Aunque la DRFQ presenta similitudes con la diabetes mellitus tipo 1 y tipo 2 , se debe considerar una entidad diferente $e^{8,9,12}$.

En los pacientes con FQ es importante el diagnóstico temprano de las alteraciones del metabolismo hidrocarbonado, puesto que el desarrollo de diabetes se asocia con un aumento de riesgo de muerte del $31-55 \%{ }^{13}$. En los pacientes con DRFQ existe un declive de la función pulmonar mayor que en los pacientes normoglucémicos, en relación directa con el grado de déficit de insulina y con la severidad de la alteración del metabolismo hidrocarbonado ${ }^{14}$. Como consecuencia, los pacientes con DRFQ tienen una menor supervivencia y una peor calidad de vida ${ }^{15}$.

Según la Guía de cuidados clínicos para DRFQ recomendada por la Asociación Americana de Diabetes $(A D A)^{16}$, los criterios diagnósticos para diabetes actualmente utilizados incluyen: glucemia en ayuno $\geq$ $126 \mathrm{mg} / \mathrm{dl}$, glucemia al azar $\geq 200 \mathrm{mg} / \mathrm{dl}$ en presencia de síntomas clásicos (poliuria y polidipsia), glucemia 2 horas de la prueba de tolerancia oral a la glucosa (PTOG) $\geq 200 \mathrm{mg} / \mathrm{dl}$ y hemoglobina glucosilada A1c $\left(\mathrm{HbA}_{1 \mathrm{C}}\right) \geq 6.5 \%$. Las tres deben ser confirmadas por repetición en días diferentes.

La HbA1c no se debería utilizar como prueba de cribado de DRFQ, porque puede ser normal en los estadios iniciales y en los casos de hiperglucemia intermitente $\mathrm{e}^{3,6,13,16,17}$.

Los niveles de glucosa plasmática, estudiados únicamente en ayunas, podrían dejar sin diagnosticar a más de dos tercios de los pacientes con DRFQ ${ }^{15,18}$.

En la actualidad la PTOG es la prueba de cribado más sensible para detectar la DRFQ y es la recomendaba por la ADA y la Sociedad Internacional de Diabetes en pediatría y adolescencia ${ }^{16,19}$. La guía de diagnóstico y tratamiento de pacientes con $\mathrm{FQ}^{1}$ recomienda realizar una PTOG anualmente a los pacientes con FQ mayores de 10 años o aquellos que presenten alguna de las siguientes situaciones: polidipsia/poliuria, fracaso en la rehabilitación nutricional a pesar de adecuadas intervenciones, baja velocidad de 
crecimiento, retraso puberal y la caída inexplicable de la función pulmonar.

La historia natural de la diabetes en la FQ sigue una evolución en el tiempo caracterizada, en primer lugar, por la aparición de hiperglucemia posprandial intermitente, seguida de intolerancia a la glucosa (ITG), DRFQ sin glucemia basal alterada (GBA) y, por último, DRFQ con GBA. Aproximadamente el 15\% de los adultos con FQ presentan diabetes relacionada a la FQ con GBA y el $25 \%$ cursan sin ella ${ }^{15,19}$.

Es importante considerar que los puntos de corte utilizados para definir DRFQ fueron tomados de los empleados para el diagnóstico de diabetes tipo 2 en la población general. Estos fueron establecidos teniendo en cuenta las complicaciones microvasculares en lugar del declive de la función pulmonar y del estado nutricional, que son las complicaciones más importantes en los pacientes con FQ. Estas complicaciones pueden preceder por años el diagnóstico de DRFQ, usando el criterio diagnóstico actual ${ }^{20}$.

Respecto de la PTOG, se ha observado que algunos sujetos con FQ sin DRFQ presentan hiperglucemias a los 30, 60 o 90 minutos, aunque los niveles a los o y 120 minutos sean similares a controles sanos. Por lo tanto, la glucemia en los puntos intermedios puede ser más sensible para detectar alteraciones del metabolismo de la glucosa en estos pacientes. Adicionalmente, estudios recientes han demostrado que existe asociación entre una peor función pulmonar y la presencia de hiperglucemia en los puntos intermedios de la PTOG ${ }^{21-23}$. La PTOG permite reconocer diferentes categorías de alteraciones del metabolismo de la glucosa (Tabla 1). Según varios estudios, con respecto a la insulina, la primera fase de la secreción en respuesta a una sobrecarga de glucosa oral e intravenosa está alterada en adultos con $\mathrm{FQ}^{6,8,14,24}$. El pico máximo de insulina está significativamente retrasado hasta los 90-120
TABLA 1. Categorías diagnósticas de las alteraciones del metabolismo hidrocarbonado según los resultados de la PTOG

\begin{tabular}{|c|c|}
\hline $\begin{array}{l}\text { Cribado y categorías } \\
\text { diagnósticas }\end{array}$ & $\begin{array}{l}\text { Resultados de la PTOG } \\
\text { (o y } 120 \mathrm{~min} \text { ) }\end{array}$ \\
\hline \multicolumn{2}{|l|}{ PTOG } \\
\hline TGN & $\mathrm{GB}<126 \mathrm{mg} / \mathrm{dl}$ y Glu-120 < $140 \mathrm{mg} / \mathrm{dl}$ \\
\hline DRFQ con GBA & $G B<126 \mathrm{mg} / \mathrm{dl}$ y Glu-120 $\geq 200 \mathrm{mg} / \mathrm{dl}$ \\
\hline DRFQ $\sin G B A$ & $G B \geq 126 \mathrm{mg} / \mathrm{dl}$ y Glu-120 $\geq 200 \mathrm{mg} / \mathrm{dl}$ \\
\hline GBA & GB $100-125 \mathrm{mg} / \mathrm{dl}$ y Glu-120 < $140 \mathrm{mg} / \mathrm{dl}$ \\
\hline ITG & 2hPTOG entre $140-199 \mathrm{mg} / \mathrm{dl}$ \\
\hline INDET & $\begin{array}{l}\text { GB }<126 \mathrm{mg} / \mathrm{dl} \text { y Glu- } 120<140 \mathrm{mg} / \mathrm{dl} \\
(\text { algún intermedio, 30, 60, } 90 \mathrm{~min} \\
\text { PTOG } \geq 200 \mathrm{mg} / \mathrm{dl} \text { ) }\end{array}$ \\
\hline $\mathrm{HbA} 1 \mathrm{c}$ & $>6,5 \%$ (valores $<6,5 \%$ no lo excluyen) \\
\hline Glucemia al azar & $\geq 200 \mathrm{mg} / \mathrm{dl}+$ poliuria + polidipsia \\
\hline \multicolumn{2}{|c|}{$\begin{array}{l}\text { TGN: tolerancia a la glucosa normal; DRFQ con GBA: diabetes relacionada } \\
\text { con fibrosis quística con glucemia basal alterada; DRFQ sin GBA: diabetes } \\
\text { relacionada con fibrosis quística sin glucemia basal alterada; GBA: glucemia } \\
\text { basal alterada; HbA1c: hemoglobina glucosilada; INDET: tolerancia a la } \\
\text { glucosa indeterminada; ITG: intolerancia a la glucosa; PTOG: prueba de } \\
\text { tolerancia oral a la glucosa; 2hPTOG: test de sobrecarga oral a la glucosa, } \\
\text { con glucemia a los o y } 120 \text { minutos. }\end{array}$} \\
\hline
\end{tabular}

minutos en pacientes con FQ, respecto a los 30-60 minutos en sujetos sanos ${ }^{10}$. Esta pérdida de la primera fase de la secreción de insulina aparece incluso en sujetos con $\mathrm{FQ}$ y una $\mathrm{TGN}^{14,24}$. La cantidad absoluta de insulina secretada a lo largo del tiempo también está disminuida en los casos de DRFQ $6,7,25,26$.

En FQ se ha comprobado el efecto beneficioso de la insulinoterapia tanto para controlar las alteraciones hidrocarbonadas como para contrarrestar el estado catabólico secundario al déficit de insulina ${ }^{26-28}$. The Cystic Fibrosis Trust en el 2004 propuso considerar el tratamiento con insulina en hiperglucemias transitorias, ITG asociada con pérdida de peso y de deterioro de las condiciones clínicas ${ }^{29}$. Investigaciones recientes sugieren el tratamiento con insulina en los pacientes con tolerancia a la glucosa indeterminada (INDET) ${ }^{30}$. La discordancia entre diagnóstico de DRFQ y el empeoramiento clínico 
puede sugerir un retraso inapropiado en la implementación de tratamiento ${ }^{31-34}$. Considerando que las alteraciones del metabolismo hidrocarbonado en pacientes con FQ no siempre se pueden evidenciar mediante las pruebas habituales de GB, PTOG o HbA1c, creemos importante estudiar los puntos intermedios de la PTOG para la detección temprana de estas y su correlación con el estado pulmonar y nutricional. Consideramos que la detección de este grupo de pacientes es importante para un control y seguimiento más estricto de las complicaciones antes mencionadas.

\section{OBJETIVO GENERAL}

Evaluar la utilidad de los puntos 30,60 y 90 minutos de la PTOG en pacientes con FQ durante la pesquisa anual de diabetes relacionada a FQ. Evaluar la correlación de las alteraciones en el metabolismo hidrocarbonado con la función pulmonar y el estado nutricional.

\section{OBJETIVOS SECUNDARIOS}

Evaluar la distribución de las alteraciones del metabolismo hidrocarbonado de acuerdo con el rango etario, como así también evaluar el comportamiento de los niveles de glucemia y de la secreción de insulina durante la PTOG.

\section{MATERIALES Y MÉTODOS}

Se llevó a cabo un estudio observacional prospectivo de corte transversal, en el que se incluyeron los pacientes mayores de 16 años con FQ que asisten al Servicio de Neumonología del Hospital Interzonal General de Agudos Dr. Rodolfo Rossi de La Plata. Los pacientes se sometieron a una PTOG extendida y evaluación clínica. Se citó a todos los pacientes que concurrieron a control al Centro de Adultos con Fibrosis Quística, que habitualmente utilizan como tratamientos solución hipertónica 7\%, dornasa alfa, azitromicina, vitaminas A, E, K y $\mathrm{D}$, ácido ursodesoxicólico, tobramicina/colistina (quienes se encuentran colonizados con Pseudomona aeruginosa), salbutamol, bromuro de ipratropio, y quienes presentan insuficiencia pancreática, lipasa-pancreatina.

Fueron considerados criterios de exclusión el diagnóstico conocido de DRFQ, glucemias basales mayores a $126 \mathrm{mg} / \mathrm{dl}$ y todas aquellas condiciones que puedan interferir significativamente con el metabolismo de la glucosa. Tales condiciones incluyen: exacerbaciones, tratamiento antibiótico intravenoso dentro del último mes, embarazo y esteroides orales. Se reprogramaron los estudios para los próximos tres meses en aquellos pacientes que presentaron signos de infección al momento de la consulta.

Se registraron los datos de genotipo asociado a la FQ y edad de cada paciente al momento del estudio.

La metodología utilizada para todos los pacientes incluyó tres etapas, que se describen a continuación.

\section{Estudios de laboratorio}

PTOG extendida y determinación de insulina:

En el laboratorio se realizó una venopunción basal. Se le indicó al paciente tomar una carga de $1.75 \mathrm{~g}$ de glucosa/kg de peso (máximo de 75 g en agua), luego de la cual se extrajeron muestras de sangre a los 30, 60, 90 y 120 minutos. 
Se determinó glucemia e insulina en las muestras extraídas a tiempo cero y a los 30, 60, 90 y $120 \mathrm{mi}$ nutos poscarga oral de glucosa. Todos los pacientes firmaron un consentimiento informado. El trabajo fue aprobado por un comité de ética.

La glucemia se realizó en equipo BT-3000 plus con reactivos, controles y calibradores Wienner lab. La técnica consiste en el ensayo colorimétrico de glucosa oxidasa-peroxidasa de punto final; se utilizaron controles Standatrol S-E niveles 1 y 2 con coeficientes de variación de 1.91 y $2.19 \%$ respectivamente. La determinación analítica de insulina se realizó en equipo Access 2 Immunoassay Systems de Beckman Coulter con reactivos y calibradores del mismo fabricante. La técnica consiste en un inmunoensayo de quimioluminiscencia tipo «sándwich»; se utilizaron controles Bio-Rad Liphochek Immunoassay Plus Control, niveles 1,2 y 3 con coeficientes de variación de $2.90,4.49$ y $3.92 \%$ respectivamente. El laboratorio participa del Programa de Evaluación Externa de la Calidad de la Fundación Bioquímica Argentina y del Ministerio de Salud de la Provincia de Buenos Aires.

Una vez analizadas las muestras se descartaron mediante los métodos habituales de bioseguridad para muestras biológicas.

\section{En el consultorio médico}

- Evaluación clínica.

- Determinaciones espirométricas: volumen espiratorio en el primer segundo $\left(\mathrm{VEF}_{1}\right)$ con espirómetro Carefusion.

- Mediciones antropométricas: peso, altura e índice de masa corporal (IMC).

\section{Análisis de datos}

Los pacientes fueron categorizados de acuerdo con la clasificación de la North American CF Consensus Conference 2010.

Se evaluó el porcentaje de alteraciones del metabolismo hidrocarbonado detectadas por la PTOG clásica y la PTOG extendida.

Se comparó estadísticamente el $\mathrm{VEF}_{1}$ y el IMC entre los pacientes con alteraciones del metabolismo hidrocarbonado y aquellos sin alteraciones.

Se evaluó la distribución de las alteraciones del metabolismo hidrocarbonado de acuerdo con distintos rangos etarios.

Se comparó el comportamiento de los niveles de glucemia y de la secreción de insulina durante la PTOG para las distintas categorías de alteraciones del metabolismo hidrocarbonado con respecto a TGN.

Los datos fueron analizados mediante el programa estadístico Graph Pad 5.0; se realizó análisis de Grubbs para detección de datos aberrantes, las comparaciones de medianas entre los grupos se realizaron mediante el test no paramétrico de Kruskal-Wallis con postest de Dunn, se consideró una $\mathrm{p}<0.05$ como estadísticamente significativa.

\section{RESULTADOS}

Se estudiaron 29 pacientes con diagnóstico de FQ durante el periodo de junio de 2016 a julio de 2017. Se excluyó del estudio a uno de ellos por presentar una glucemia basal mayor a $126 \mathrm{mg} / \mathrm{dl}$.

De los 28 pacientes incluidos en el estudio, 9 fueron mujeres (32.2\%) y 19 hombres (67.8\%). La media de edad fue de 29 años, con un rango de 16 a 65 años. 
TABLA 2. Categorías de alteraciones del metabolismo hidrocarbonado y parámetros de función pulmonar $\left(V F_{1}, V F_{1} \%\right)$ y del estado nutricional (IMC)

\begin{tabular}{|l|c|c|c|c|}
\hline & TGN $(\mathbf{n}=14)$ mediana $(D E)$ & ITG $(n=7)$ mediana $(D E)$ & GBA $(n=3)$ mediana $(D E)$ & INDET $(n=4)$ mediana $(D E)$ \\
\hline $\operatorname{VEF}_{1}$ (litros) & $3.08(1.14)$ & $1.31(0.75)^{*}$ & $1.61(1.37)$ & $2.28(0.55)$ \\
\hline $\operatorname{VEF}_{1} \%(\%)$ & $80(25.18)$ & $44.5(22.55)$ & $41(26.21)$ & $54(19.91)$ \\
\hline $\mathrm{IMC}\left(\mathrm{kg} / \mathrm{m}^{2}\right)$ & $24.45(3.60)$ & $21.1(2.29)$ & $22.7(1.21)$ & $22.3(2.76)$ \\
\hline
\end{tabular}

*Diferencias significativas para VEF, con respecto a TGN ( $p<0.05$, prueba de Dunn).

IMC: índice de masa corporal; TCN: tolerancia a la glucosa normal; ITG: intolerancia a la glucosa; GBA: glucemia basal alterada; INDET: tolerancia a la glucosa indeterminada; $\mathrm{VEF}$; volumen espiratorio forzado en el primer segundo; $\mathrm{VEF} \%$ : volumen espiratorio forzado porcentual.

En cuanto al genotipo, 13 pacientes (46.43\%) presentaron la mutación DF508 heterocigota, 3 (10.71\%) DF508 homocigota, 8 (28.57\%) presentaron otras mutaciones y de 3 pacientes se desconoce el genotipo.

Los pacientes estudiados fueron categorizados mediante el resultado de la PTOG. Se encontraron: 14 (50\%) con TGN, $3(10,7 \%)$ con GBA, 7 (25\%) con ITG y $4(14.3 \%)$ con INDET. El porcentaje de pacientes con alteraciones del metabolismo hidrocarbonado detectado por medio de la PTOG clásica fue del 35.7\% $(n=10)$ y por la PTOG extendida de $50 \%(n=14)$. A los cuatro años de seguimiento los cuatro pacientes que presentaron prueba con INDET evolucionaron diabetes relacionada a la FQ.

Con respecto a la evaluación del $\mathrm{VEF}_{1}$, se encontraron diferencias significativas entre los grupos ITG y TGN, mientras que no se encontraron diferencias significativas para los grupos INDET y GBA en relación con la TGN. La evaluación del VEF \% y el IMC no mostró diferencias significativas entre los grupos. Los resultados obtenidos se muestran en la tabla 2 y la figura 1. La distribución de las distintas alteraciones metabólicas según edad se muestra en la figura 2. A partir de los resultados de la PTOG extendida, el análisis de comparación de medianas mostró diferencias significativas para la glucemia basal entre GBA y TGN, para la glucemia a los 30 minutos entre INDET y TGN, y para las glucemias a los 60, 90 y 120 minutos entre ITG y TGN. No se encontraron diferencias significativas para la insulinemia entre los grupos en ninguno de los puntos de la curva (Tabla 3). Las curvas de glucemia e insulinemia se observan en la figura 3.

\section{DISCUSIÓN}

En nuestro estudio evaluamos la PTOG extendida en pacientes con FQ mayores de 16 años incluyendo los puntos de 30, 60 y 90 minutos a la PTOG clásica. Encontramos que la mitad de los pacientes presentaron alteraciones del metabolismo hidrocarbonado, sin tener en cuenta aquellos con GB mayor a 126 $\mathrm{mg} / \mathrm{dl}$ o diagnóstico conocido de DRFQ. Si consideramos solo los puntos intermedios de la curva, detectamos un $14.3 \%$ de individuos con glucemias mayores a $200 \mathrm{mg} / \mathrm{dl}$ (INDET). Los resultados por Cano Megías, et al. ${ }^{2}$ son similares a los encontrados por nosotros. Otros autores encontraron un porcentaje menor de INDET, que se puede justificar porque emplearon únicamente el punto intermedio de los 60 minutos de la PTOG ${ }^{35}$. Si bien algunos investigadores sugieren el punto de los 60 minutos como el más sensible para detectar alteraciones del metabolismo hidrocarbonado, en nuestro estudio detectamos INDET en todos los puntos intermedios de la PTOG. Creemos que la importancia de estos hallazgos es que este grupo de pacientes no serían diagnosticados empleando como tamizaje de DRFQ a la PTOG clásica ${ }^{21,36}$. Concluimos que el empleo de 
A

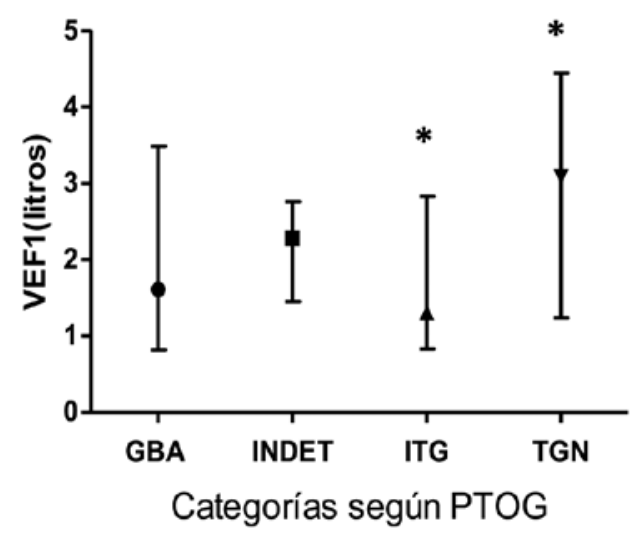

B

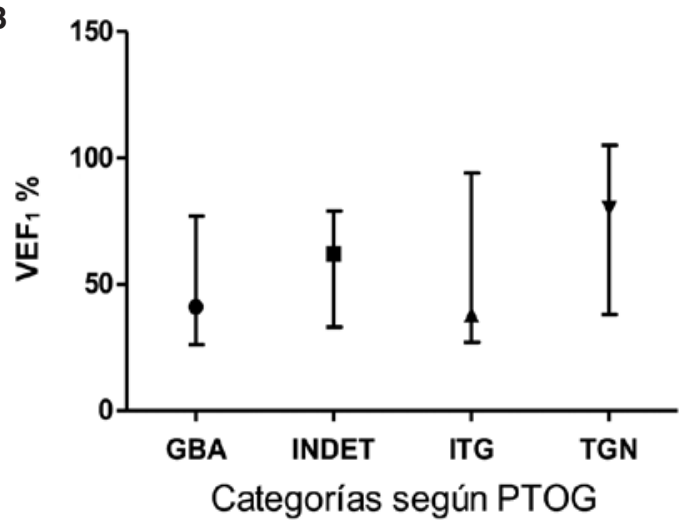

C

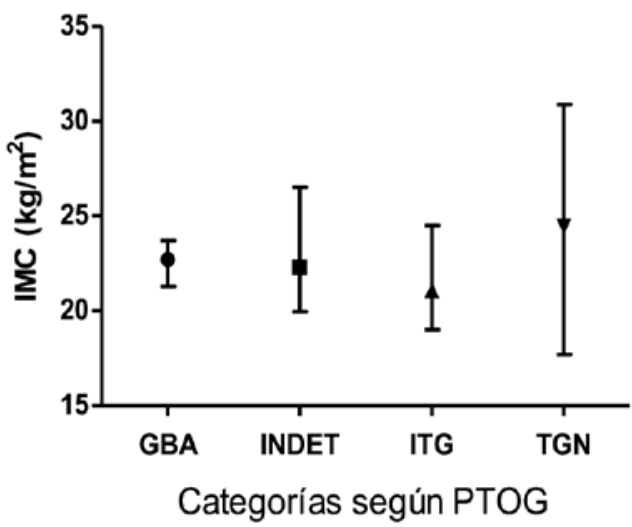

Figura 1. Función pulmonar y estado nutricional en pacientes con fibrosis quística. Comparación de los parámetros de función pulmonar y nutricional entre las distintas categorías de alteraciones del metabolismo hidrocarbonado. Mediana ( $\pm \mathrm{DE}$ ) de $\mathrm{VEF}_{1}$ en litros $(\mathbf{A}), \mathrm{VEF}_{1} \%(\mathbf{B})$ e IMC en $\mathrm{kg} / \mathrm{m}^{2}$ (C), para GBA (n=3), INDET ( $=4)$, ITG ( $n=7)$ y TGN ( $n=14)$.

*Diferencias significativas para $\mathrm{VEF}_{1}$ con respecto a TGN ( $\leftarrow \leftarrow 0.05$, prueba de Dunn).

PTOG: prueba de tolerancia oral a la glucosa; TGN: tolerancia a la glucosa normal; GBA: glucemia basal alterada; INDET: tolerancia a la glucosa indeterminada; ITG: intolerancia a la glucosa; VEF ${ }_{1}$ : volumen espiratorio forzado en el primer segundo; $V_{E} F_{1} \%$ : volumen espiratorio forzado porcentual; IMC: índice de masa corporal.

la PTOG extendida permite la detección temprana de un mayor número de pacientes con alteraciones del metabolismo hidrocarbonado.

Con respecto a la evaluación de la función pulmonar basada en el $V E F_{1}$, el grupo de ITG fue el único que evidenció un declive de esta en relación con la TGN. En contraste con otros autores, no encontramos alteraciones de la función pulmonar en los pacientes INDET34,37. Estos hallazgos nos hacen pensar que al momento del diagnóstico aún no presentaban alteraciones pulmonares. Sin embargo, en nuestro trabajo este grupo está constituido por un número de individuos muy bajo como para inferir un comportamiento. Sin embargo, todos aquellos que obtuvieron valores indeterminados en la prueba, cuatro años más tarde evolucionaron a DRFQ. Además, Coriati, et al. ${ }^{34}$ estudiaron una población pediátrica que presentaba un mayor porcentaje del genotipo homocigota DF508 (46.4\%), mientras que en nuestro estudio incluimos pacientes mayores de 16 años y este genotipo representó solo un $10.71 \%$. En relación con la 


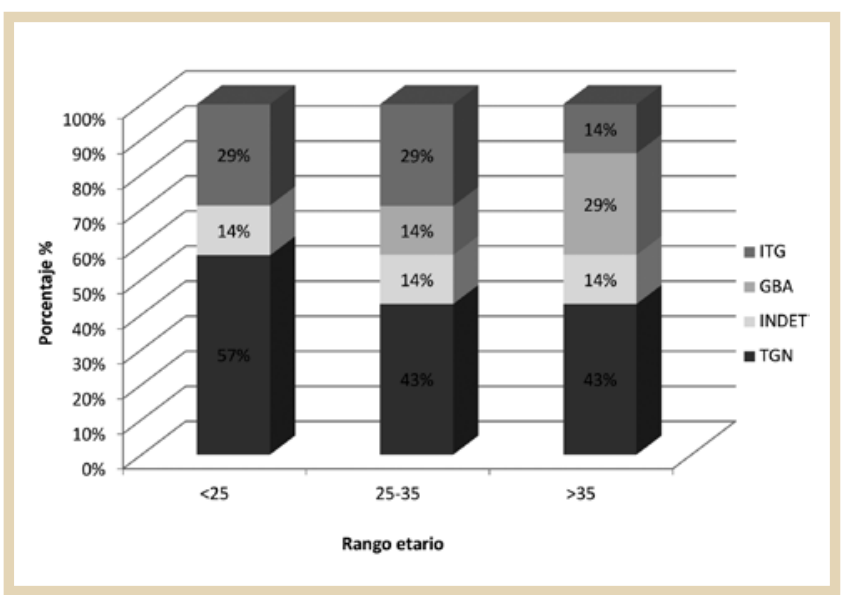

Figura 2. Categorías de las alteraciones del metabolismo hidrocarbonado de acuerdo con la PTOG expresadas en porcentaje para cada grupo etario. PTOG: prueba de tolerancia oral a la glucosa; TGN: tolerancia a la glucosa normal; GBA: glucemia basal alterada; INDET: tolerancia a la glucosa indeterminada; ITG: intolerancia a la glucosa.

función nutricional evaluada con el IMC, no vimos diferencias significativas entre los distintos grupos, a diferencia de otros autores que demostraron un peor estado nutricional en pacientes ITG 34,38 .

Según la bibliografía consultada, se conoce que los pacientes con DRFQ presentan una enfermedad pulmonar más severa y peor estado nutricional comparado con los pacientes con FQ sin diabetes ${ }^{7,25,38}$. Esto hace suponer que la detección de alteraciones tempranas del metabolismo hidrocarbonado permitiría la implementación de medidas preventivas con el fin de retrasar el desarrollo de diabetes y las complicaciones antes mencionadas. Existen evidencias de que este grupo de pacientes podría beneficiarse del tratamiento con insulina, observándose aumento del $V F_{1}$ y mejora del estado nutricional ${ }^{30,31,39-41}$.

En cuanto a la observación de las curvas durante la PTOG extendida, se destaca que en el punto de los 30 minutos, los pacientes INDET presentaron el pico máximo de glucemia, con valores significativamente mayores a los del grupo TGN. En relación con la insulinemia, a diferencia de otros autores, nuestro grupo de trabajo no encontró un retraso en el pico de insulina ${ }^{2,34}$. El comportamiento de la glucosa podría deberse a una respuesta retrasada a la secreción de insulina en los pacientes INDET, que se explicaría por la resistencia a la insulina que presentan estos individuos ${ }^{14,42,43}$. Por otro lado, en concordancia con los estudios de Hardin y Moran ${ }^{44}$, observamos progresión de las alteraciones del metabolismo de los hidratos de carbono con la edad. Encontramos que más del $50 \%$ de los pacientes menores de 25 años presentaban tolerancia normal a la glucosa al momento del estudio. En cambio, en el grupo de mayor edad, el $57 \%$ de los pacientes presentó algún tipo de alteración. En todos los rangos de edad estudiados se encontraron pacientes INDET.

Debido a la detección de pacientes INDET mediante la PTOG extendida en todo el rango de edad estudiado, creemos recomendable el empleo de esta en el tamizaje anual de los pacientes con FQ mayores de 16 años de edad. Se necesitan más estudios para trasladar esta sugerencia a los sujetos menores de 16 años, dado que no fueron incluidos en nuestra investigación.

\section{CONCLUSIONES}

Mediante el empleo de la PTOG extendida en individuos con $\mathrm{FQ}$, se detectó un $14.3 \%$ de pacientes INDET, los cuales se hubiesen perdido al realizar la PTOG clásica. Estos sujetos no presentaron alteraciones pulmonares ni nutricionales en comparación con el grupo TGN al momento del estudio.

Se observó un aumento de las alteraciones del metabolismo hidrocarbonado con la edad, encontrándose INDET en todo el rango de edades estudiado. 
TABLA 3. Glucemia e insulinemia durante la PTOG extendida para las distintas categorías de alteraciones del metabolismo hidrocarbonado

\begin{tabular}{|l|l|c|c|c|c|}
\hline Tiempo (minutos) & & $\begin{array}{c}\text { TGN }(\mathbf{n}=14) \\
\text { mediana (DE) }\end{array}$ & $\begin{array}{c}\text { ITG }(\mathbf{n}=7) \\
\text { mediana (DE) }\end{array}$ & $\begin{array}{c}\text { GBA }(\mathbf{n}=3) \\
\text { mediana (DE) }\end{array}$ & $\begin{array}{c}\text { INDET (n=4) } \\
\text { mediana (DE) }\end{array}$ \\
\hline \multirow{2}{*}{0} & Glucemia & $88.5(11.06)$ & $94.0(9.76)$ & $106.0(2.08)^{*}$ & $98.0(5.96)$ \\
\cline { 2 - 6 } & Insulinemia & $5.4(1.82)$ & $3.7(1.80)$ & $3.7(0.76)$ & $7.0(3.99)$ \\
\hline \multirow{2}{*}{30} & Glucemia & $151.5(25.91)$ & $164.0(28.40)$ & $168.0(42.75)$ & $212.0(33.39)^{*}$ \\
\cline { 2 - 6 } & Insulinemia & $39.6(33.30)$ & $23.3(8.80)$ & $30.9(27.08)$ & $53.0(15.70)$ \\
\hline \multirow{2}{*}{60} & Glucemia & $151.0(31.79)$ & $193.0(9.31)^{*}$ & $162.0(41.64)$ & $185.0(20.96)$ \\
\cline { 2 - 6 } & Insulinemia & $52.9(32.48)$ & $35.7(21.88)$ & $64.2(30.65)$ & $54.0(18.77)$ \\
\hline \multirow{2}{*}{120} & Glucemia & $113.5(32.49)$ & $194.0(8.53) *$ & $102.0(28.94)$ & $105.0(8.06)$ \\
\cline { 2 - 6 } & Insulinemia & $37.8(40.72)$ & $50.3(23.28)$ & $32.0(6.00)$ & $29.3(15.07)$ \\
\hline & Glucemia & $99.0(35.57)$ & $164.5(26.79)^{*}$ & $80.0(5.13)$ & $65.0(11.62)$ \\
\cline { 2 - 6 } & Insulinemia & $26.1(30.34)$ & $48.4(40.24)$ & $14.0(13.91)$ & $17.8(13.06)$ \\
\hline
\end{tabular}

*Diferencias significativas para glucemia con respecto a TGN ( $p<0.05$, prueba de Dunn).

PTOG: prueba de tolerancia oral a la glucosa; TGN: tolerancia a la glucosa normal; ITG: intolerancia a la glucosa; GBA: glucemia basal alterada; INDET: tolerancia a la glucosa indeterminada.
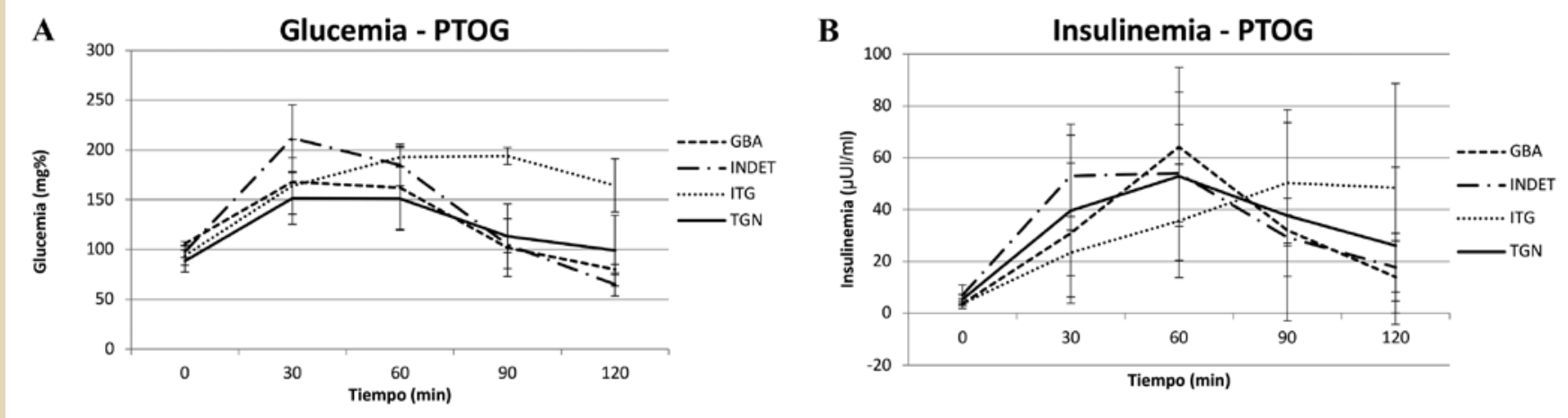

Figura 3. Curvas de glucemia e insulinemia durante la PTOG en pacientes con fibrosis quística. Mediana ( \pm DE) de: concentración de glucosa plasmática (mg/dl) (A) y concentración de insulina plasmática ( $\mu U l / m l)$ (B), luego de la PTOG en pacientes con fibrosis quística para las distintas categorías de alteraciones del metabolismo hidrocarbonado. PTOG: prueba de tolerancia oral a la glucosa; TGN: tolerancia a la glucosa normal; ITG: intolerancia a la glucosa; GBA: glucemia basal alterada; INDET: tolerancia a la glucosa indeterminada.

Los pacientes INDET fueron detectados en todos los puntos intermedios de la PTOG extendida, presentando un pico máximo de glucemia a los 30 minutos. Por su parte, el comportamiento de la insulina plasmática no mostró diferencias entre los grupos estudiados.

En conclusión, el empleo de la PTOG extendida permite la detección temprana de un mayor número de pacientes con alteraciones del metabolismo hidrocarbonado.

\section{FINANCIAMIENTO}

La presente investigación no ha recibido ninguna beca específica de agencias de los sectores públicos, comercial o sin ánimo de lucro. 


\section{CONFLICTOS DE INTERESES}

Los autores declaran no tener conflictos de intereses.

\section{RESPONSABILIDADES ÉTICAS}

\author{
Protección de personas y animales. Los autores de- \\ claran que para esta investigación no se han realiza- \\ do experimentos en seres humanos ni en animales.
}

\section{Confidencialidad de los datos. Los autores declaran} que han seguido los protocolos de su centro de trabajo sobre la publicación de datos de pacientes.

\section{Derecho a la privacidad y consentimiento informa- \\ do. Los autores declaran que en este artículo no aparecen datos de pacientes.}

\section{BIBLIOGRAFÍA}

1. Elston C, Simmonds N, Geddes D. The future for a child born with cystic fibrosis today. Breathe. 2007;4:16-23.

2. Cano Magías M, Gonzalez Albarran O, Guisado Vasco P, Lamas Ferreiro A, Máiz Carro L. Resistencia insulínica, disfunción de la célula $\beta$ pancreática y diferencias en los puntos intermedios de las curvas de glucemia e insulina tras una sobrecarga oral estándar de glucosa en pacientes con fibrosis quística. Endocrinol Nutr. 2015;62:91-9.

3. Nathan BM, Laguna T, Moran A. Recent trends in cystic fibrosis-related diabetes. Curr Opin Endocrinol Diabetes Obes. 2010;17(4):335-41.

4. Ortigoza L. Fibrosis quística. Aspectos diagnósticos. Colomb Med. 2007;38(1 Supl 1):41-9.

5. O'Riordan SMP, Dattani MT, Hindmarsh PC. Cystic fibrosis-related diabetes in childhood. Horm Res Pedriatr. 2010;73:15-24.

6. Moran A, Hardin D, Rodman D, Allen HF, Beall RJ, Borowitz D, et al. Diagnosis, screening, and management of cystic fibrosis related diabetes mellitus. A consensus conference report. Diabetes Res Clin Pract. 1999;45:61-73.

7. Moran A, Dunitz J, Nathan B, Saeed A, Holme B, Thomas W. Cystic fibrosis-related diabetes: current trends in prevalence, incidence, and mortality. Diabetes Care. 2009;32(9):1626-31.

8. Lanng S. Glucose intolerance in cystic fibrosis patients. Paediatric Respir Rev. 2001;2:253-9.

9. Laguna TA, Nathan BM, Moran A. Managing diabetes in cystic fibrosis. Diabetes Obes Metab. 2010;12(10):858-64.

10. Malay R, Craig FM, Selvadurai H, Donaghue KC, Craig ME. Cystic fibrosis-related diabetes in children - Gaps in the evidence? Nat Rev Endocrinol. 2010;6:371-8.

11. Lanng S, Thorsteinsson B, Nerup J, Koch C. Diabetes mellitus in cystic fibrosis: effect of insulin therapy on lung function and infections. Acta Paediatr. 1994;83:849-85.
12. Ntimbane T, Comte B, Mailhot G, Berthiaume Y, Poitout V, Prentki M, et al. Cystic fibrosis-related diabetes: From CFTR dysfunction to oxidative stress. Clin Biochem Rev. 2009;30(4):153-77.

13. Chamnan P, Shine BS, Haworth CS, Bilton D, Adler Al. Diabetes as a determinant of mortality in cystic fibrosis. Diabetes Care. 2010;33(2): 311-6.

14. Alves CAD, Aguiar RA, Alves ACS, Santana MA. Diabetes mellitus in patients with cystic fibrosis. J Bras Pneumol. 2007;33(2):213-21.

15. Cano Megías M, González Albarrán O. Diabetes en la fibrosis quística: una entidad diferente. Endocrinol Nutr. 2015;62(1):38-44.

16. Moran A, Brunzell C, Cohen RC, Katz M, Marshall BC, Onady G, et al. Clinical care guidelines for cystic fibrosis-related diabetes. A position statement of the American Diabetes Association and a clinical practice guideline of the Cystic Fibrosis Foundation, endorsed by the Pediatric Endocrine Society. Diabetes Care. 2010;33(12).

17. Acerini C, Craig ME, de Beaufort C, Maahs DM, Hanas R. Introduction to ISPAD Clinical Practice Consensus Guidelines 2014 Compendium. Pediatr Diabetes. 2014;15(Suppl 20):1-3.

18. Lanng S, Hansen A, Thorsteinsson B, Nerup J, Koch C. Glucose tolerance in patients with cystic fibrosis: five year prospective study. BMJ. 1995;311:655-9.

19. O'Riordan SM, Robinson PD, Donaghue KC, Moran A. Management of cystic fibrosis-related diabetes. Pediatr Diabetes. 2008;9(4 Pt 1):338-44.

20. Hameed S, Jaffé A, Verge CF. Advances in the detection and management of cystic fibrosis related diabetes. Curr Opin Pediatr. 2015;27(4):525-33.

21. Schmid K, Fink K, Holl RW, Hebestreit H, Ballmann M. Predictors for future cystic fibrosis-related diabetes by oral glucose tolerance test. J Cyst Fibros. 2014;13(1):80-5.

22. Nyboe Andersen B, Krarup T, Thorsgaard Pedersen NT, Faber OK, Hagen C, Worning H. B cell function in patients with chronic pancreatitis and its relation to exocrine pancreatic function. Diabetologia. 1982;23(2):86-9.

23. Lee KM, Miller RJ, Rosenberg FM, Kreisman SH. Evaluation of glucose tolerance in cystic fibrosis: comparison of 50-g and 75-g tests. J Cyst Fibros. 2007;6(4):274-6.

24. O'Riordan SM, Robinson PD, Donaghue KC, Moran A. Management of cystic fibrosis-related diabetes in children and adolescents. Pediatr Diabetes. 2009;10(Supl. 12):43-50.

25. Marshall BC, Butler SM, Stoddard M, Moran AM, Liou TG, Morgan WJ. Epidemiology of cystic fibrosis-related diabetes. J Pediatr. 2005;146(5): 681-7.

26. Moran A, Becker D, Casella SJ, Gottlieb PA, Kirkman MS, Marshall BC, et al. Epidemiology, pathophysiology, and prognostic implications of cystic fibrosis-related diabetes: a technical review. Diabetes Care. 2010;33(12):2677-83.

27. Martín-Frías M, Lamas Ferreiro A, Enes Romeroa P, Cano Gutiérrez B, Barrio Castellanos R. Alteraciones hidrocarbonadas en pacientes impúberes con fibrosis quística. An Pediatr (Barc). 2012;77(5):339-43.

28. Mohan K, Israel KL, Miller H, Grainger R, Ledson MJ, Walshaw MJ. Long-term effect of insulin treatment in cystic fibrosis-related diabetes. Respiration. 2008;76(2):181-6.

29. Cystic Fibrosis our focus. Management of Cystic Fibrosis Related Diabetes Mellitus [Internet]. The UK Cystic Fibrosis Trust Working Group; junio de 2004. Disponible en: https://www.cysticfibrosis.org.uk/sites/ default/files/2020-12/Diabetes\%20mellitus\%2omanagement\%20 Jun\%2004.pdf

30. Donaghue K, Robinson PD. Cystic fibrosis-related diabetes mellitus [Internet]. Uptodate; junio 2017. Disponible en: https://www.uptodate.com/contents/cystic-fibrosis-related-diabetes-mellitus

31. Bizzarri C, Lucidi V, Ciampalini P, Bella S, Russo B, Cappa M. Clinical effects of early treatment with insulin glargine in patients with cystic fibrosis and impaired glucose tolerance. J Endocrinol Invest. 2006;29:RC1-4. 
32. Sheikh S, Kelly A. Cystic fibrosis-related diabetes: links, challenges, and future directions. Review. Res Rep Endocr Disor. 2015;5:157-67.

33. Tofé S, Moreno JC, Maiz L, Alonso M, Escobar H, Barrio R. Insulin secretion abnormalities and clinical deterioration related to impaired glucose tolerance in cystic fibrosis. Eur J Endocrinol. 2005;152(2):241-7.

34. Coriati A, Ziai S, Azar M, Berthiaume Y, Rabasa-Lhoret R. Characterization of patients with cystic fibrosis presenting an indeterminate glucose tolerance (INDET). J Cyst Fibros. 2016;15(1):127-32.

35. Noronha RM, Damaceno N, Muramatu LH, Monte O, Calliari LEP. Importance of screening with oral glucose tolerance test for early diagnosis of cystic fibrosis-related diabetes mellitus. Pediatr Diabetes. 2014;15(4):309-12.

36. Ode KL, Frohnert B, Laguna T, Phillips J, Holme B, Regelmann W, et al. Oral glucose tolerance testing in children with cystic fibrosis. Pediatr Diabetes. 2010;11(7):487-92.

37. Brodsky J, Dougherty S, Makani R, Rubenstein RC, Kelly A. Elevation of 1-hour plasma glucose during oral glucose tolerance testing is associated with worse pulmonary function in cystic fibrosis. Diabetes Care. 2011;34(2):292-5.

38. Milla CE, Warwick WJ, Moran A. Trends in pulmonary function in patients with cystic fibrosis correlate with the degree of glucose intolerance at baseline. Am J Respir Crit Care Med. 2000;162:891-5.
39. Hameed S, Morton JR, Field PI, Belessis Y, Yoong T, Katz T, et al. Once daily insulin detemir in cystic fibrosis with insulin deficiency. Arch Dis Child. 2012;97(5):464-7.

40. Mozzillo E, Franzese A, Valerio G, Sepe A, De Simone I, Mazzarella G, et al. One-year glargine treatment can improve the course of lung disease in children and adolescents with cystic fibrosis and early glucose derangements. Pediatr Diabetes. 2009;10(3):162-7.

41. Koloušková S, Zemková D, Bartošová J, Skalická V, Šumník Z, Vávrová $\checkmark$, et al. Low-dose insulin therapy in patients with cystic fibrosis and early-stage insulinopenia prevents deterioration of lung function: a 3-year prospective study. J Pediatr Endocrinol Metab. 2011;24(7-8): 449-54.

42. Hardin DS, Leblanc A, Marshall G, Seilheimer DK. Mechanisms of insulin resistance in cystic fibrosis. Am J Physiol Endocrinol Metab. 2001;281(5):1022-8.

43. Battezzati A, Mari A, Zazzeron L, Alicandro G, Claut L, Battezzati PM, et al. Identification of insulin secretory defects and insulin resistance during oral glucose tolerance test in a cohort of cystic fibrosis patients. Eur J Endocrinol. 2011;165(1):69-76.

44. Hardin DS, Moran A. Diabetes mellitus in cystic fibrosis. Endocrinol Metab Clin North Am. 1999; 28:787-800. 